\title{
Review on Sustained Relief of Osteoarthritis Symptoms with a Proprietary Extract from Pine Bark, Pycnogenol
}

\author{
Peter Jörg Rohdewald \\ Institute for Pharmaceutical and Medicinal Chemistry, Westfälische Wilhelms-Universität, Münster, Germany.

\begin{abstract}
This review summarizes the effects of the standardized proprietary bark extract of the French maritime pine $\left(\right.$ Pycnogenol ${ }^{\circledR}$ ) in mild osteoarthritis (OA), stage 1 and 2. The extract exerts antioxidative, anti-inflammatory, and chondroprotective effects in vitro and in vivo. Its phenolic acids as well as catechin and taxifolin are quickly absorbed. Active metabolites, produced by gut microbiota in the intestinal tract from oligomeric procyanidins, appear in blood $6 \mathrm{~h}$ following ingestion and remain for at least $14 \mathrm{~h}$, providing a long-lasting flow of anti-inflammatory substances for relief of OA symptoms. These constituents of Pycnogenol could be detected in serum, blood cells, and synovial fluid of OA patients. The resulting inhibition of cartilage-destructing proteases and pain-producing cyclo-oxygenases provides the basis for relief from pain, improvement of stiffness, enhanced mobility, and well-being in three clinical studies with the pine bark extract as an adjunct supplement. Sparing the use of nonsteroidal anti-inflammatory drugs, supplementation with the pine bark extract reduced gastric complications and hospital admissions of OA patients. Because of its favorable safety profile and sustained
\end{abstract} \\ anti-inflammatory action, Pycnogenol represents an option as an add-on supplement for OA patients.
}

KEYWORDS: maritime pine extract USP $\bullet$ matrix metalloproteases $\bullet$ procyanidins $\bullet$ osteoarthritis $\bullet$ taxifolin $\bullet$ WOMAC scores

\section{INTRODUCTION}

$\mathbf{O}$ STEOARTHRitis (OA) IS PREDOMINANTLY a disease of the elderly, affecting primarily the knees and hips. As OA progresses with age, the worldwide increasing life expectancy leads to an enormous burden for healthcare in many countries.

Symptoms of OA, such as reduced mobility of joints and pain, may be reduced for the time being by weight loss, exercise, and physiotherapy. The high prevalence of synovitis in OA contributes substantially to the development of inflammation and pain. ${ }^{1}$ One pharmacological approach attempts to supplement damaged cartilage with its constituents chondroitin, hyaluronic acid, and glucosamines. By far the most widespread symptomatic treatment of $\mathrm{OA}$ is the oral or local application of steroidal or nonsteroidal anti-inflammatory drugs (NSAIDs) to fight the chronic local inflammation and pain.

The long-term use of oral NSAIDs may lead to serious unwanted effects, as there is a fivefold increased risk for

\footnotetext{
Manuscript received 30 January 2017. Revision accepted 20 July 2017.
}

Address correspondence to: Prof. Dr. Peter Jörg Rohdewald, Institute of Pharmaceutical and Medicinal Chemistry, Westfälische Wilhelms-Universität, Twenteweg 15, Münster 48161, Germany, E-mail: rohdewa@uni-muenster.de

(C) Peter Jörg Rohdewald, 2018; Published by Mary Ann Liebert, Inc. This is an Open Access article distributed under the terms of the Creative Commons Attribution License, which permits unrestricted use, distribution, and reproduction in any medium, provided the original work is properly cited. peptic ulcers and upper gastrointestinal injuries such as bleeding up to perforation in the intestinal tract. ${ }^{2}$ Furthermore, NSAIDs are associated with renal adverse effects, high doses may cause kidney failure. ${ }^{2}$ Especially a triple therapy with NSAIDs and ACE inhibitors or angiotensin receptor blockers with diuretics is at risk for acute kidney injury. ${ }^{3}$ Hence, there is a need to substitute or to reduce the use for NSAIDs by supplementation with a safe standardized plant extract. ${ }^{4}$ Furthermore, the use of NSAIDs over a prolonged period of time may increase blood pressure. ${ }^{4}$

\section{PYCNOGENOL $^{\circledR}$}

\section{Composition}

Pycnogenol, a standardized extract from the bark of the French maritime pine (Pinus pinaster Aiton), consists of a concentrate of polyphenols. Main components with 60-75\% oligomeric are procyanidins besides taxifolin, catechin, and phenol acids. ${ }^{5}$ The extract, distributed worldwide by Horphag Research (UK) Ltd, corresponds to the standard of the US Pharmacopeia (USP 39). ${ }^{6}$

\section{Sustained release}

Ideally, the persistent inflammation causing permanent pain should not be treated by using pain killers occasionally, but by a preparation providing a constant anti-inflammatory action. For this purpose, the pharmaceutical industry provides 
sustained release formulations of NSAIDs. Unfortunately, the permanent release of NSAIDs produces not only pain relief for a long period of time but also a permanent gastrointestinal irritation.

\section{Pharmacokinetics}

Pycnogenol mimics a sustained release formulation not by release of active components from a matrix, but by its natural combination of short- and long-acting anti-inflammatory substances. Some components of Pycnogenol such as catechin, ferulic acid, and caffeic acid are found rapidly in plasma after $30 \mathrm{~min}$, with peak concentrations between 1 and $4 \mathrm{~h}$, taxifolin appears later in plasma with a maximum at $8 \mathrm{~h}^{7}$

Even at $14 \mathrm{~h}$, catechin, taxifolin, and ferulic acid are present in plasma. Ferulic acid could be found in urine up to $25 \mathrm{~h}$ after ingestion. ${ }^{8}$

The active metabolite M1, a valerolactone, appears in plasma not before $6 \mathrm{~h}$ with peak concentrations at $10 \mathrm{~h}$. M1 concentrations are still present after $14 \mathrm{~h}^{7}$

This pharmacokinetic profile of M1 is consistent with the bacterial metabolization of the main constituents of Pycnogenol, the oligomeric procyanidins. The oligomers consist of catechin and epicatechin units, forming chains from dimers up to 12 monomeric units. ${ }^{5}$

In the first step of metabolization, the monomers catechin or epicatechin are split from the oligomers, and subsequently, bacterial activity transforms the monomers into derivatives of $\delta$-valerolactone, the metabolites M1 and M2. ${ }^{9}$ As Pycnogenol consists between $65 \%$ and $75 \%$ of oligomeric procyanidins, the formation of the M1 may proceed over several hours because of the stepwise separation of the monomeric units from the pool of oligomers by the activity of the intestinal bacteria. ${ }^{5}$ This secondary metabolization produces a prolonged flow of anti-inflammatory substances in addition to the anti-inflammatory phenolic constituents of Pycnogenol.

As all of these constituents of Pycnogenol and its metabolites exhibit anti-inflammatory actions, the progressing appearance of the diverse active substances provides a longlasting pain relief, so that patients feel less pain, also during the night. ${ }^{10}$

Hence, Pycnogenol's long-lasting action is not based on sustained release from a formulation, but by the successive appearance of its diverse anti-inflammatory constituents and metabolites in blood stream.

\section{Anti-inflammatory activity}

The constituents of Pycnogenol act in concert, as all of its phenolic compounds are scavengers of free radicals and exhibit a range of anti-inflammatory actions, as documented for ferulic acid, caffeic acid, catechin, and taxifolin. ${ }^{3,8-14}$

The metabolites, formed by ring fission of catechin units by microbiota, possess also remarkable anti-inflammatory activity. The procyanidin metabolite M1 showed in vitro a $100 \%$ higher activity than hydrocortisone. ${ }^{15}$ These initial in vitro effects could be proven in ex vivo experiments.

Plasma collected from volunteers subsequent to consumption of Pycnogenol, inhibited the activity of cycloox- ygenases 1 and 2, as well as the activation of the inflammation "master switch" nuclear factor kappa B $(\mathrm{NF} \kappa \mathrm{B}) .{ }^{15}$

Human pharmacokinetic investigations indicated that the metabolite M1 is enriched in blood cells by active transport mechanisms, so that anti-inflammatory activity of blood is most probably 30 times higher than measured in plasma. ${ }^{16}$

Furthermore, the active metabolite M1, ferulic acid, and caffeic acid are present in the synovial fluid, thus acting directly on the source of inflammation in case of synovitis. ${ }^{17}$

\section{PRECLINICAL INVESTIGATION}

\section{Protection of articular cartilage}

The progression of $\mathrm{OA}$ is connected with a blockage of synthesis of proteoglycan components and type 2 collagen by inflammatory cytokines and tumor necrosis factor alpha. ${ }^{18-20}$ Furthermore, cartilage degrading proteolytic enzymes such as matrix metalloproteinases (MMPs) are liberated as MMP-1, MMP-2, and MMP-13. ${ }^{21}$ The metabolite M1 formed from the procyanidins of Pycnogenol inhibited the activity of MMP-1, MMP-2, and MMP-9 in vitro and blocked the release of MMP-9 from activated monocytes. ${ }^{15}$ These chondroprotective effects were confirmed in ex vivo experiments. Plasma, taken from human volunteers following intake of Pycnogenol, inhibits the release of $\mathrm{NF} \kappa \mathrm{B}$ and MMP-9 from activated monocytes. The inhibition of the master switch of inflammation $\mathrm{NF} \kappa \mathrm{B}$, acting together with inflammatory cytokines, reduces considerably the inflammatory process connected to OA. Analysis of synovial fluid from OA patients revealed the presence of ferulic acid, caffeic acid, taxifolin, catechin, and the metabolite M1 in serum, blood cells, and synovial fluid. ${ }^{17}$ The anti-inflammatory substances ferulic acid, caffeic acid, and the active metabolite M1 were enriched in the synovial fluid relative to serum. So, the chondroprotective and anti-inflammatory action take place locally in the synovia. The chondroprotective action of Pycnogenol could be demonstrated in patients with sever OA (Jessberger et al., unpublished). Chondrocytes, isolated from cartilage postoperatively, express significantly less cartilage-destructing enzymes, as the matrix metalloproteases MMP-3 and MMP-13 as well as the inflammatory IL-1 $\beta$. In serum, levels of aggrecanases, which destroy the cartilage building block aggrecan, were significantly lowered (Jessberger et al., unpublished).

The reduced anti-inflammatory activity in OA with Pycnogenol is reflected in a decrease of C-reactive protein levels in OA patients by $70 \%$, with plasma-free radicals simultaneously scavenged by $30 \% .^{22}$

The cooperation of the diverse anti-inflammatory effects of Pycnogenol in plasma and synovial fluid results in reduction of pain and increased mobility in clinical studies cited below.

\section{CLINICAL EFFICACY TRIALS}

Three identically designed clinical trials investigating the role of Pycnogenol in OA treatment have been published to date. $10,23,24$ 
Table 1. Overview of Three Clinical Trials Demonstrating Efficacy of Pycnogenol for Arthritis

\begin{tabular}{|c|c|c|c|}
\hline \multirow[b]{3}{*}{$\%$ improvement } & Belcaro et al. ${ }^{22}$ & Cisar et al. ${ }^{24}$ & Farid et al. ${ }^{23}$ \\
\hline & $\mathrm{N}=156$ & $\mathrm{~N}=100$ & $\mathrm{~N}=35$ \\
\hline & $100 \mathrm{mg} /$ day & $150 \mathrm{mg} / \mathrm{day}$ & $150 \mathrm{mg} /$ day \\
\hline Pain & $-45 \%$ & $-40 \%$ & $-43 \%$ \\
\hline Stiffness & $-53 \%$ & $-40 \%$ & $-35 \%$ \\
\hline Physical performance & $+56 \%$ & $+22 \%$ & $+52 \%$ \\
\hline Global score & $+50 \%$ & & $+49 \%$ \\
\hline
\end{tabular}

All studies were randomized, double blind, and placebo controlled. Middle-aged patients (48-54 years) suffering from mild OA, stage I or II, verified by X-ray, were treated either with $3 \times 50 \mathrm{mg}$ Pycnogenol daily or placebo, added to existing therapy with NSAIDs. Success of the add-on supplementation was objectivated by the Western Ontario McMasters University (WOMAC) questionnaire for OA during a period of 3 months. Results are summarized in Table 1.

The first, small-scale study $(n=35)$ showed a clear reduction of scores for pain, functionality, and total WOMAC score, dependent on duration of treatment. ${ }^{23}$ After 3 months, significant $(P<.001)$ differences to placebo were observed, symptoms were reduced by $43 \%, 35 \%, 52 \%$, and $49 \%$ for pain, stiffness, physical function, and total WOMAC score, respectively.

In the second study, involving 100 patients, the pain score improved significantly $(P<.004)$ with time compared to baseline. ${ }^{24}$ In this study, a considerable placebo effect was observed for pain, daily activity scores, and overall WOMAC scores, and although improvement relative to baseline was highly significant $(P<.001-P<.03)$, the difference to placebo did not reach significance level in any case. Only scores for stiffness improved significantly both to baseline $(P<.01)$ and versus placebo $(P<.05)$ after 2 and 3 months, while placebo had no effect.

A total of 156 patients were included in the third study. ${ }^{10}$ Symptom scores dropped significantly: pain by $45 \%$, stiffness by $47 \%$, physical function by $43 \%$, and overall WOMAC score by $44 \%$. The decrease under placebo was not significant. A more detailed analysis of the WOMAC scores of the third study revealed a significant reduction $(P<.05)$ of nocturnal pain and pain during troublesome stair climbing, particularly relevant for the quality of life of OA patients. ${ }^{10}$

Also, for joint stiffness during the day, only minor changes were observed with placebo, while Pycnogenol improved stiffness remarkably $(P<.05)$.

An example for an enhanced physical function refers to the onerous rising from sitting: with Pycnogenol the score dropped clearly from initial 3.1 to 0.8 , with placebo the score remained nearly unchanged (3.0 to 3.1).

The improvement of OA was impressively objectivated by performance of patients on a treadmill. Patients in the Pycnogenol group could increase their walking distance after 3 months from 68 to $198 \mathrm{~m}$, whereas placebo expanded walking distance from $65 \mathrm{~m}$ just to $88 \mathrm{~m} .{ }^{10}$

These positive effects related to relief from daily pain, stiffness, and physical function had of course a great influ- ence on the well-being of the patients in the Pycnogenol group. As the negative impact of $\mathrm{OA}$ on daily activities subsided, the emotional status of patients shifted significantly $(P<.05)$ from irritability, frustration, depression, and insomnia to better well-being. ${ }^{10}$ The sum of negative emotional scores dropped from 31.4 to 11.5 , whereas placebo had a nonsignificant effect (28.4 to 24.1). Thus, quality of life was definitely improved in the Pycnogenol group.

It has to be emphasized that patients were allowed to use their NSAIDs as concomitant medication when needed.

Use of NSAIDs was significantly reduced in all three studies in the Pycnogenol group, in contrast to a slight increase of intake of NSAIDs in the placebo group. A more precise evaluation of NSAID use was performed in the third study. ${ }^{10}$

Intake of Pycnogenol allowed patients to decrease intake of NSAID medication by $58 \%$ according to their diaries. ${ }^{10}$ Correspondingly, gastrointestinal complications decreased by $63 \%$ as well as days spent in hospital (60\%). Values under placebo decreased by only $1 \%$ (reduction of use of NSAIDs) and 3\% (reduction of hospital admissions and gastrointestinal problems).

Together, the three clinical studies demonstrate a highly significant improvement of symptoms of mild OA under Pycnogenol, despite the reduced intake of NSAIDs. No unwanted effects of Pycnogenol were reported in the three studies. This is in line with the safety profile of Pycnogenol. Unwanted effects such as headache, dizziness, nausea, sleepiness, skin irritation, and gastric troubles were mild with a rate of $1.9 \%$ in clinical trials involving 7000 patients. ${ }^{25}$

\section{CONCLUSION}

Pycnogenol acts like a sustained-release formulation by its combination of fast absorbed phenolic compounds and slowly metabolized procyanidins. Its constituent ferulic acid and the metabolite M1 are enriched in synovial fluid and contribute to local anti-inflammatory action.

Pycnogenol as an anti-inflammatory and chondroprotective add-on supplement provided long-lasting positive effects such as enhanced physical mobility and pain relief for patients with mild OA. The use of NSAIDs could be significantly reduced, thus diminishing unwanted effects of NSAIDs. Studies involving more patients are needed to confirm the beneficial actions of Pycnogenol on an even broader basis.

\section{AUTHOR DISCLOSURE STATEMENT}

P.J.R. is a retired director of R\&D for Horphag Research Ltd., working now as a scientific consultant.

\section{REFERENCES}

1. Mathiessen A, Conaghan PG: Synovitis in osteoarthritis: Current understanding with therapeutic implications. Arthritis Res Ther 2017;19:18.

2. Gooch K, Culleton BF, Manns BJ, et al:: NSAID use and progression of chronic kidney disease. Am J Med 2007;120: 280.e1-e7. 
3. Lapi F, Azoulay L, Suissa S: Author's reply to Moore. BMJ 2013;346:f1016.

4. Snowden S, Nelson R: The effects of nonsteroidal antiinflammatory drugs on blood pressure in hypertensive patients. Cardiol Rev 2011;19:184-191.

5. Rohdewald P: A review of the French maritime pine bark extract $\left(\right.$ Pycnogenol ${ }^{\circledR}$ ), a herbal medication with a diverse clinical pharmacology. Int J Clin Pharmacol Ther 2002;40:158-168.

6. Maritime Pine Extract: USP edition 39. United States Pharmacopeial Convention, Inc., Rockville. In: United States Pharmacopoeia, 2016, pp. 6748-6749.

7. Grimm T, Skrabala R, Chovanova Z, et al.: Single and multiple dose pharmacokinetics of maritime pine bark extract (Pycnogenol) after oral administration to healthy volunteers. BMC Clinical Pharmacol 2006;6:4.

8. Canali R, Comitato R, Schonlau F, et al.: The anti-inflammatory pharmacology of Pycnogenol in humans involves COX-2 and 5LOX mRNA expression in leukocytes. Int Immunopharmacol 2009;9:1145-1149.

9. Grosse-Düweler K, Rohdewald P: Urinary metabolites of French maritime pine bark extract in humans. Pharmazie 2000;55: 364-368.

10. Belcaro G, Cesarone MR, Errichi S, et al.: Treatment of osteoarthritis with Pycnogenol ${ }^{\circledR}$. The SVOS (San Valentino Osteoarthritis Study). Evaluation of signs, symptoms, physical performance and vascular aspects. Phytother Res 2008;22:518523.

11. Mancuso C, Santangelo R: Ferulic acid: Pharmacological and toxicological aspects. Food Chem Toxicol 2014;65:185-195.

12. Habtemariam S: Protective effects of caffeic acid and the Alzheimer's brain: An update. Mini Rev Med Chem 2017;17: 667-674.

13. Abd El-Aziz TA, Mohamed RH, Pasha HF, Abdel-Aziz HR: Catechin protects against oxidative stress and inflammatorymediated cardiotoxicity in adriamycin-treated rats. Clin Exp Med 2012;12:233-240.

14. Kim J, Lee Y, An H-J, Lee J-D, Yi Y: Anti-inflammatory activities of taxifolin from Opuntia humifusa in lipopolysaccharide stimulated RAW 264.7 murine macrophages. Appl Biol Chem 2015;58:241-246.

15. Grimm T, Chovanova Z, Muchova J, et al: Inhibition of NF- $\kappa \mathrm{B}$ activation and MMP-9 secretion by plasma of human volunteers after ingestion of maritime pine bark extract (Pycnogenol). J Inflamm 2006;3:1.

16. Kurlbaum M, Mülek M, Högger P: Facilitated uptake of a bioactive metabolite of maritime pine bark extract (Pycnogenol) into human erythrocytes. PLoS One 2013;8:1-10.

17. Mülek M, Seefried L, Genest F, et al.: Distribution of constituents and metabolites of maritime pine bark extract (Pycnogenol) into serum, blood cells, and synovial fluid of patients with severe osteoarthritis: A randomized controlled trial. Nutrients 2017;9:443.

18. Wojdasiewicz P, Poniatowski LA, Szukiewicz D: The role of inflammatory and anti-inflammatory cytokines in the pathogenesis of osteoarthritis. Mediators Inflamm 2014;2014:561459.

19. Saklatvala J: Tumor necrosis factor alpha stimulates resorption and inhibits synthesis of proteoglycan in cartilage. Nature 1986; 322:547-549.

20. Seguin CA, Bernier SM: TNF alpha suppresses link protein and type II collagen expression in chondrocytes role of MEK1/2 and NF-kappaB signaling pathways. J Cell Physiol 2003;197: 356-369.

21. Daheshia M, Yao JQ: The interleukin 1beta pathway in the pathogenesis of osteoarthritis. J Rheumatol 2008;35:2306-2312.

22. Belcaro G, Cesarone MR, Errichi S, Zulli C, Errichi BM, Vinciguerra G, et al.: Variations in C-reactive protein, plasma free radicals and fibrinogen values in patients with osteoarthritis treated with Pycnogenol ${ }^{\circledR}$. Redox Rep 2008;13:271-276.

23. Farid R, Mirfeizi Z, Mirheidari M, et al:: Pycnogenol supplementation reduces pain and stiffness and improves physical function in adults with knee arthritis. Nutr Res 2007;27:692-697.

24. Cisar P, Jany R, Waczulikova I, et al.: Effect of pine bark extract (Pycnogenol ${ }^{\circledR}$ ) on symptoms of knee osteoarthritis. Phytother Res 2008;22:1087-1092.

25. Rohdewald P: Update on the clinical pharmacology of Pycnogenol $^{\circledR}$. Med Res Arch 2015;3:1-11. 